\title{
Análisis de las propiedades psicométricas de la Utrecht Work Engagement Scale en una muestra de trabajadores en Puerto Rico*
}

\author{
Analysis of Psychometric Properties of the Utrecht Work \\ Engagement Scale in a Sample of Workers in Puerto Rico
}

Recibido: octubre 14 de 2013 | Revisado: marzo 14 de 2014 | Aceptado: marzo 14 de 2014

\author{
RAMÓN RODRÍGUEZ-MONTALBÁN ** \\ Miguel MARTÍNEZ-LUGO ***** \\ Universidad Carlos Albizu, San Juan, Puerto Rico \\ ISRAEL SÁNCHEZ-CARDONA **** \\ Universitat Jaume I, Castellón, España
}

doi:10.11144/Javeriana.UPSY13-4.appu

Para citar este artículo: Rodríguez-Montalbán, R., Martínez-Lugo, M., Sánchez-Cardona, I.(2014) Análisis de las propiedades psicométricas de la Utrecht Work Engagement Scale en una muestra de trabajadores en Puerto Rico. Universitas Psychologica, 13(4), 1255-1266. http://dx.doi.org/10.11144/ Javeriana.UPSY13-4.appu

Agradecemos a los estudiantes graduados del Programa de Psicología Industrial/Organizacional de la Universidad Carlos Albizu, por su colaboración en esta investigación.

** Profesor adjunto del Programa de Psicología Industrial/Organizacional y miembro del Centro Institucional de Investigación Científica (CIIC)

Correo electrónico: rmontalban@sunmail.albizu. edu*** israel.sanchez1@upr.edu

****** Correo electrónico: mmartinez@albizu.edu

\section{RES UMEN}

El estudio del engagement en el trabajo es cada vez más común en la literatura científica. La estructura factorial del instrumento más utilizado por los investigadores para evaluar el engagement, la Utrecht Work Engagement Scale (UWES), ha mostrado múltiples inconsistencias en diversos países. El objetivo de este trabajo es examinar las propiedades psicométricas de la UWES en una muestra de 2.796 trabajadores en Puerto Rico. Se examinó la estructura factorial de la escala mediante ecuaciones estructurales, con el método de estimación de máxima verosimilitud. Los resultados muestran que el modelo de tres factores posee un mejor ajuste a los datos de la escala, siendo consistente con la concepción del engagement como constructo compuesto por tres dimensiones: vigor, dedicación y absorción. La validez y la fiabilidad de la UWES son adecuadas, por lo que se concluyó que la escala posee las cualidades para ser utilizada en investigaciones en el contexto laboral puertorriqueño.

Palabras clave

Engagement en el trabajo; análisis confirmatorio de factores; validez de constructo; análisis confiabilidad; Utrecth Work Engagement Scale

\section{A B S T R A C T}

The study of work engagement is becoming increasingly common in the scientific literature. There are many inconsistencies regarding the factorial structure of the Utrecht Work Engagement Scale (UWES) the most widely used instrument by researchers in different countries. The objective of this study is to examine the psychometric properties of the UWES using a sample of 2,796 employees in Puerto Rico. We examined the psychometric properties of UWES using structural equations with the method of maximum likelihood estimation. The results show that the three-factor model has a better fit, being consistent with the conception of engagement as a construct formed by three dimensions: vigor, dedication, and absorption. The validity and reliability of UWES are appropriate, and we conclude that the scale has the qualities to be used in research in the Puerto Rican labor context. Keywords

Work engagement; confirmatory factor analysis; construct validity; reliability analysis; Utrecth Work Engagement Scale 


\section{Introducción}

En los últimos años, el estudio psicológico del trabajo y las organizaciones enfocó su mirada en el desarrollo de las capacidades y las fortalezas de las personas, los grupos y las organizaciones para lograr un funcionamiento óptimo, especialmente en tiempos de crisis económicas y sociales (Salanova, Llorens, Cifre, \& Martínez, 2012; Salanova, Martínez, \& Llorens, 2005; Seligman \& Csiksentmihalyi, 2000). Este giro hacia la psicología positiva, en general, y a la psicología organizacional positiva, en específico, llevó al estudio y desarrollo de instrumentos para la evaluación de actitudes y experiencias positivas en el trabajo, como es el caso del engagement en el trabajo.

El engagement en el trabajo es un constructo motivacional positivo relacionado con el trabajo, que se caracteriza por el vigor, la dedicación y la absorción (Schaufeli, Salanova, González-Romá, $\&$ Bakker, 2002). El vigor implica altos niveles de energía y resistencia mental mientras se trabaja, por el deseo de esforzarse en el trabajo que se está realizando, incluso cuando se presentan dificultades. La dedicación consiste en una alta implicación laboral junto con la manifestación de un sentimiento de significación, entusiasmo, inspiración, orgullo y reto por el trabajo. Por último, la absorción se produce cuando la persona está totalmente concentrada en su trabajo, cuando el tiempo le pasa rápidamente y presenta dificultades a la hora de desconectarse de lo que se está realizando, debido al alto nivel de disfrute y concentración que experimenta. El engagement, más que un estado específico y momentáneo, es un estado afectivo-cognitivo más persistente y no focalizado en un objeto, evento o situación particular.

Dada la importancia que representa el engagement como un estado motivacional y de bienestar positivo para las personas trabajadoras y para las organizaciones, resulta importante su evaluación en los esfuerzos organizacionales, para atender los factores psicosociales. Para el desarrollo de organizaciones saludables, es preciso continuar evaluando y previniendo riesgos psicosociales prevalentes en las organizaciones como el estrés, el burnout, el mob- bing, entre otros, pero también resulta imprescindible examinar y potenciar las fortalezas y los estados de bienestar como el engagement, la satisfacción y las emociones positivas. En Latinoamérica existe una amplia tradición en el estudio del estrés y riesgos físicos en el trabajo (Andrade \& Gómez, 2008; Gómez, 2007), pero solo recientemente hemos ido integrando a nuestros estudios y repertorios de trabajo aspectos desde la psicología organizacional positiva (Gómez, 2007; Sanín \& Salanova, 2014).

Ante tal panorama, resulta importante desarrollar una agenda de investigación desde la psicología positiva, dentro del área de la psicología del trabajo y las organizaciones. El desarrollo de esta agenda investigativa requiere de instrumentos válidos y confiables para la evaluación de constructos como el engagement en el trabajo en contextos latinoamericanos. Este estudio pretende ofrecer evidencia empírica de las propiedades psicométricas de la Utrecht Work Engagement Scale (UWES), con una muestra de trabajadores en Puerto Rico, a fin de contribuir al desarrollo del estudio de las fortalezas y el bienestar en los contextos de trabajo en Puerto Rico y Latinoamérica.

\section{Engagement en el trabajo: un breve trasfondo y su importancia para las organizaciones}

El concepto de engagement tomó prominencia en la literatura sobre el trabajo y las organizaciones cuando Kahn (1990) teorizó por primera vez sobre el engagement en el trabajo y describió a las personas engaged como aquellas que están física, cognitiva y emocionalmente conectados con sus roles en el trabajo. La propuesta de Kahn estaba dirigida a lo que definió como engagement personal; es decir, se centró en la forma en que las personas satisfacen sus roles de trabajo y asumió que las personas engaged se esfuerzan mucho en su trabajo, porque se identifican con él y se definen a sí mismos en términos de sus roles de trabajo (Salanova \& Schaufeli, 2009). Posteriormente, el engagement se integra al estudio del síndrome de quemarse por el trabajo (burnout) en las investigaciones de Maslach y Leiter (1997) y Maslach, Schaufeli y Leiter (2001). Estos trabajos 
sirven de antesala a las distintas conceptuaciones del constructo de engagement, así como con implicaciones para su medición y evaluación.

Una de las primeras conceptuaciones del engagement lo propone como la antítesis o el opuesto del burnout. Esta primera mirada al constructo, presentada por Maslach y Leiter (1997), caracteriza al engagement en el trabajo por su energía, involucramiento y eficacia, las cuales precisamente son las tres dimensiones opuestas del burnout: agotamiento, cinismo y falta de eficacia profesional. Desde esta perspectiva, se presume que el burnout y el engagement son dos polos opuestos de un mismo continuo. El burnout se describe entonces como una erosión del engagement en el trabajo, donde la energía se torna en agotamiento, el involucramiento en cinismo y la eficacia en falta de eficacia profesional. Desde esta línea de investigación, se utiliza el Maslach Burnout Inventory General Survey ([MBI-GS]; Maslach, Jackson, \& Leiter, 1996) y más recientemente otros instrumentos como el Oldelburg Burnout Inventory (Demerouti \& Bakker, 2008; Halbesleben \& Demerouti, 2005), para medir el engagement en el trabajo, partiendo de que ambos constructos son los opuestos de un contínuo, donde bajas puntuaciones en estas escalas son indicadores de engagement.

Una línea posterior del estudio del engagement reconoce, al igual que la anterior, que el engagement en el trabajo es el opuesto al burnout; sin embargo, define, explica y mide al engagement en el trabajo de forma propia e independiente. Esta perspectiva es la que propone Schaufeli et al. (2002) al definir el engagement como un concepto multidimensional caracterizado por el vigor, la dedicación y la absorción. Esta nueva perspectiva requería el desarrollo de un instrumento de medición que fuera cónsono con la idea propuesta. De ahí surge la Utrecht Work Engagement Scale (UWES) como respuesta para el aporte a un instrumento que midiera el engagement como un constructo distinto, aunque relacionado al burnout.

No se puede dejar de mencionar que aun cuando existen investigaciones que puntualizan la independencia de ambos constructos, tanto para su conceptuación como para su medición (e. g., Demerouti,
Mostert, \& Bakker, 2010; González-Romá, Schaufeli, Bakker, \& Lloret, 2006), estudios recientes realizan fuertes críticas a la idea de diferenciación entre burnout y engagement, alegando que existe redundancia en ambos constructos (Cole, Walter, Bedeian, \& O’Boyle, 2012). No obstante, la mayor parte de la evidencia empírica apoya la idea de que son constructos distintos y la UWES continua siendo el cuestionario más utilizado para medirlo (Salanova \& Schaufeli, 2009).

\section{Importancia del engagement para el trabajo y las organizaciones}

En la última década, la investigación del engagement ha demostrado la importancia del mismo para los individuos, los equipos y las organizaciones. La relación entre el engagement y los resultados individuales y organizacionales positivos está representada en el modelo de demandas-recursos laborales (Job Demands-Resources Model [DRL]). Este modelo trata de explicar el engagement y el burnout en función de las demandas (personales, laborales y organizacionales) y los recursos (personales, laborales y organizacionales) y sus consecuencias a nivel individual y organizacional (Bakker, 2011; Bakker $\&$ Demerouti, 2008; Demerouti, Bakker, Nachreiner, \& Schaufeli, 2001). El modelo DRL intenta explicar el bienestar y el malestar de los trabajadores a través del proceso motivacional y de deterioro de la salud. En el proceso motivacional, las personas pueden balancear las demandas del trabajo con los recursos tanto personales como organizacionales, generando resultados positivos tales como el engagement y el desempeño favorable. El modelo sostiene que a través de este proceso motivacional, integrado por el engagement, los resultados a nivel organizacional son positivos: mayor productividad y buen desempeño.

Por otro lado, para explicar el malestar, el modelo propone un proceso de deterioro de la salud. En este proceso, cuando las demandas laborales son mayores que los recursos laborales y personales, el resultado corresponde a mayores niveles de burnout. Esto trae consigo efectos nocivos para las personas trabajadoras tales como el agotamiento 
emocional, la despersonalización y la falta de eficacia personal. De esta forma, se producen resultados organizacionales negativos como menor productividad, mayor estrés y pobre desempeño (i. e., Hakanen, Shcaufeli, \& Ahola, 2008; Llorens, Bakker, Schaufeli, \& Salanova, 2006; Lorente, Salanova, Martínez, \& Schaufeli, 2008).

Siguiendo la línea del modelo de demandas y recursos laborales, la investigación muestra evidencia de la relación del engagement con resultados individuales y organizacionales positivos como la satisfacción laboral (Salanova, Schaufeli, Llorens, Peiro, \& Grau, 2000), el compromiso organizacional y la involucración en el trabajo (Halberg \& Schaufeli, 2006), las conductas intrarrol y extrarrol (Kim, Kolb, \& Kim, 2013), la iniciativa personal (Salanova \& Schaufeli, 2009), el desempeño individual y grupal (Salanova, Augut, \& Peiró, 2005; Torrente, Salanova, Llorens, \& Schaufeli, 2012) e incluso el desempeño financiero de las organizaciones (Xanthopoulou, Bakker, Demerouti, \& Schaufeli, 2009). De igual modo, la investigación reciente ha identificado algunos antecedentes del desarrollo de engagement entre los cuales destacan los recursos personales (i. e., autoeficacia, competencias mentales y emocionales) (Lorente et al., 2008), recursos y prácticas laborales (i. e., desarrollo de carrera, desarrollo de habilidades, prácticas de salud psicosocial, trabajo en equipo) (Acosta, Torrente, Llorens, \& Salanova, 2013; Torrente et al., 2012), así como el rol del liderazgo, del apoyo y de la participación en la toma de decisiones (Salanova, Lorente, Chambel, \& Martínez, 2011; Román, Battistelli, \& Odoardi, 2013).

\section{Medición y evaluación del engagement: Utrecht Work Engagement Scale}

\footnotetext{
La Utrecht Work Engagement Scale (UWES) es un instrumento desarrollado para evaluar al engagement según la propuesta de Schaufeli et al. (2002), donde se describe como un estado afectivo motivacional caracterizado por el vigor, la dedicación y la absorción. La versión en inglés la desarrollaron Schaufeli et al. (2002), mientras que en español la desarrollaron Salanova et al. (2000). Esta escala es
}

la más utilizada para examinar el engagement en el trabajo y se ha traducido a 17 idiomas.

La escala original de la UWES estaba compuesta por 24 ítems que intentaban reflejar las tres dimensiones del constructo (vigor, dedicación y absorción) (Schaufeli et al., 2002). Posteriormente, los autores redujeron la escala a una versión de 17 ítems para lograr una medición más parsimoniosa. Esta versión del instrumento está compuesta de tres subescalas: seis ítems de vigor, cinco ítems de dedicación y seis ítems de absorción (Schaufeli et al., 2002). Luego Schaufeli, Bakker y Salanova (2006) utilizaron los datos recopilados en 10 países $(n=$ 14.521) para desarrollar una versión reducida de la escala, que consta de nueve ítems agrupados en tres factores: tres ítems de vigor, tres ítems de dedicación y tres ítems de absorción (UWES-9, por sus siglas en inglés). De acuerdo con Schaufeli et al. (2006), este instrumento es el más utilizado en la investigación del engagement en distintos países del mundo. En Latinoamérica, la escala se ha utilizado en varias investigaciones, encontrando evidencia positiva y significativa entre el engagement en el trabajo y la implicación laboral (Martínez-Lugo et al., 2011), el clima organizacional (Martínez-Lugo, 2009) y la interacción positiva trabajo-familia y la interacción positiva familia-trabajo (Rodríguez-Montalbán, Vélez-Pastrana, \& Meléndez, 2012).

\section{Estudios de las propiedades psicométricas del UWES}

A pesar de ser un concepto relativamente nuevo dentro del campo de la psicología, el engagement ha sido ampliamente estudiado, tomando mayor prominencia los estudios realizados en Europa, Asia y más recientemente en los Estados Unidos y Latinoamérica. Dada la importancia de su estudio y de las propiedades psicométricas derivadas del instrumento desarrollado por Schaufeli et al. (2002), varios investigadores en diversos países le han prestado importancia a la investigación de sus propiedades psicométricas, a saber: Holanda (Schaufeli et al., 2002), España (Salanova, et al., 2000), Portugal (Schaufeli, Martínez, et al., 2002), Sur África (Storm \& Rothmann, 2003), Japón (Shimazu et al., 2008), 
China (Fong \& Ng, 2012), Finlandia (Seppälä et al., 2009), Noruega (Nerstad, Richardsen, \& Martinussen, 2010), Italia (Balducci, Fraccaroli, \& Schaufeli, 2010), Estados Unidos (Mills, Culbertson, \& Fullagar, 2011; Muilenberg-Trevino, 2009; Wefald \& Downey, 2009) y Argentina (Spontón, Medrano, Maffei, Spontón, \& Castellano, 2012).

Es importante destacar que pese a que se han realizado múltiples estudios sobre las propiedades psicométricas de la UWES, los hallazgos son un tanto inconsistentes en cuanto a su estructura factorial. Varios de estos estudios han demostrado que la estructura factorial, tanto de la UWES-9 como de la UWES-17, está compuesta de tres factores altamente correlacionados: vigor, dedicación y absorción (Balducci et al., 2010; Nerstad et al., 2010; Salanova et al., 2000; Schaufeli et al., 2002; Schaufeli et al., 2006). Mientras que otros estudios han encontrado un solo factor (Muilenburg-Treviño, 2009; Sëppäla et al., 2009; Shimazu et al., 2008).

A esto se añade la escasa evidencia científica sobre la UWES en países latinoamericanos, donde a nuestro conocimiento, solo se destaca el estudio realizado por Spontón et al. (2012) en Argentina, quienes encontraron una estructura factorial de dos y tres factores para este instrumento. A pesar de esta inconsistencia, en múltiples estudios ha quedado demostrado que la UWES- 9 y la UWES-17 poseen altos índices de fiabilidad: vigor $(\alpha=0.73$. 0.91), dedicación ( $\alpha=0.83-0.92)$ y absorción ( $\alpha$ $=0.69-0.87$ ).

La inconsistencia sobre la estructura factorial de la UWES plantea la siguiente pregunta: ¿Cuál es la estructura factorial de la UWES en una muestra de trabajadores de Puerto Rico? Tomando como base este interrogante, el objetivo de este estudio es analizar las propiedades psicométricas de la versión en español de la UWES-9 en un grupo de personas trabajadoras en Puerto Rico.

\section{Método}

\section{Participantes}

Para este estudio, e utilizaron las bases de datos de distintas investigaciones realizadas en Puerto
Rico donde se incluyó la variable engagement en el trabajo. Todos los datos que componen la muestra provienen de diferentes estudios en los que los investigadores han participado. La muestra final está compuesta por 2.796 participantes. Como criterio de inclusión para esta investigación, los participantes tenían que ser mayores de 21 años y al momento del estudio, estar trabajando. La muestra estuvo compuesta mayoritariamente por hombres (56.7 \%). Del mismo modo la mayoría de los participantes laboraban en el sector privado (74.5 \%) y sus edades oscilaban entre 21 y 77 años $(M=36.99 ; D E=10.43)$.

\section{Instrumentos}

Para recopilar los datos de esta investigación se utilizaron los siguientes instrumentos:

\section{Cuestionario de información demográfica}

A través del cual se obtuvo la información referente al sexo, edad, preparación académica, estado civil y tipo de organización donde se trabaja.

Versión en español del Utrecht Work Engagement Scale ([UWES]; Schaufeli, Bakker, Eु Salanova, 2006)

Este instrumento consta de nueve ítems distribuidos en tres ítems para cada dimensión del engagement (vigor, dedicación y absorción), con una escala tipo Likert de siete anclajes los cuales comienzan desde nunca o ninguna vez, hasta siempre o todos los días. Los índices de fiabilidad alfa de Cronbach son los siguientes: vigor $(\alpha=0.82)$, dedicación $(\alpha=0.86)$ y absorción $(\alpha=0.8)$.

\section{Procedimiento para los análisis de datos}

Una vez recopilada la información de las distintas bases de datos, se procedió a unificarlos en una sola plantilla de datos en el programa SPSS 21. Para cumplir con el objetivo del estudio, se realizaron análisis descriptivos (medias y desviaciones 
estándar), análisis de distribución de los datos (Kolmogorov-Smirnov y Shapiro-Wilk), análisis de factores confirmatorios, análisis de fiabilidad y correlaciones. Para el análisis de factores confirmatorio se empleó el programa AMOS 21 (Arbuckle, 2011) con el método de estimación de máxima verosimilitud y el programa $R$ para realizar las correcciones de Satorra y Bentler (2001) para los estadísticos de ajuste de modelos de ecuaciones estructurales con datos no normales. Se usaron las correcciones de ajuste de modelos propuestas por Satorra y Bentler (2001) para los índices de chicuadrado, Índice de Ajuste Normativo de Bentler y Bonnet (Normed Fit Index [NFI]), Índice de Ajuste No Normativo (Non-Normed Fit Index [NNFI]) y el Índice de Ajuste Comparativo (Comparative Fit Index [CFI]). También se incluyeron en el estudio los índices de raíz media cuadrática de residuales (Standardized Root Mean Square Residual [SRMR]) y el Akaike Information Criterion ([AIC]; Akaike, 1987) para comparar modelos competitivos no anidados.

Se analizaron dos modelos competitivos de ecuaciones estructurales: un modelo de un solo factor (M1), donde todos los ítems cargaban a un factor latente, y un modelo de tres factores (M2) propuestos por Schaufeli et al. (2002). Una vez analizada la estructura factorial que presentó los mejores índices de ajuste, se procedió a hacer un análisis de discriminación de los ítems, a través de la correlaciónítem-total. Enseguida, se calculó la fiabilidad de la escala, mediante el coeficiente de fiabilidad alfa de Cronbach, así como la fiabilidad compuesta de los factores latentes, siguiendo las recomendaciones de
Raykov y Shrout (2002). Finalmente, se procedió a examinar la validez convergente y discriminante de los tres factores, siguiendo las recomendaciones de Fornell y Larcker (1981), a través del cálculo de la varianza media extraída (VME).

\section{Resultados}

La Tabla 1 muestra los estadísticos descriptivos (medias y desviaciones estándar), alfa de Cronbach, fiabilidad compuesta, varianza media extraída y correlaciones.

Se calcularon las medias y desviaciones estándar para cada ítem del UWES. Las medias de los ítems fluctuaron entre 4.08 y 5.16, y las desviaciones estándar fluctuaron entre 1.21 y 1.67. En la Tabla 2 se observa que las pruebas Kolmogorov-Smirnov y Shapiro-Wilk ofrecen evidencia empírica de que los datos no siguen una distribución normal. El estadístico de estimación normalizada de la curtosis multivariada, coeficiente de Mardia $=169.812, p<$ 0.001 , justifica la utilización del método estimación de máxima verosimilitud con estimación robusta (Mardia, 1970).

Con base en hallazgos previos, se pusieron a prueba dos modelos, un modelo de tres factores de engagement (vigor, dedicación y absorción) propuestos por Schaufeli et al. (2002) y un modelo unifactorial, partiendo de los hallazgos de otros estudios (Muilenburg-Treviño, 2009; RodríguezMontalbán, 2011; Shiamatzu et al., 2008; Sonnetang, 2003). En la Tabla 2, se muestran los indicadores de ajuste para los modelos puestos a prueba. Los indicadores de ajuste del modelo de un factor

TABLA 1

Medias, desviaciones estándar, alfas, fiabilidad compuesta, varianza media extraída y correlaciones $(n=2.796)$

\begin{tabular}{lcccccccc}
\hline & $\mathrm{M}$ & $\mathrm{DE}$ & $\alpha$ & $\mathrm{FC}$ & $\mathrm{VME}$ & 1 & 2 & 3 \\
\hline Vigor & 4.53 & 1.21 & 0.82 & 0.83 & 0.62 & & 0.93 & 0.88 \\
Dedicación & 4.75 & 1.28 & 0.86 & 0.87 & 0.69 & 0.8 & & 0.87 \\
Absorción & 4.51 & 1.28 & 0.8 & 0.81 & 0.59 & 0.72 & 0.75 & \\
Engagement & 4.6 & 1.15 & 0.92 & 0.85 & 0.63 & 0.92 & 0.93 & 0.9 \\
\hline
\end{tabular}

Nota. Todas las correlaciones son significativas a $p<0.01 ; \mathrm{M}=$ media; $\mathrm{DE}=$ desviación estándar; $\alpha=$ alfa de Cronbach; FC = fiabilidad compuesta; VME = varianza media extraída. Los valores sobre la diagonal representan las correlaciones de entre los factores latentes, mientras que los valores debajo de la diagonal representan las correlaciones de las puntuaciones directas. Fuente: elaboración propia 
TABLA 2

Indicadores de ajuste para análisis confirmatorio de factores

\begin{tabular}{ccccccccccc}
\hline Modelo & $\chi 2$ corr & GL & $p$ & NFIcorr & NNFIcorr & CFIcorr & SRMR & AIC & $\Delta \chi 2$ corr & $\Delta$ AIC \\
\hline M1 & 697.07 & 27 & 0 & 0.87 & 0.91 & 0.92 & 0.04 & 1210.4 & & \\
M2 & 467.06 & 24 & 0 & 0.92 & 0.93 & 0.95 & 0.03 & 830.47 & & \\
Dif. M1- M2 & & & & & & & & & $230.01 * *$ & 379.93 \\
\hline
\end{tabular}

Nota. $\mathrm{M} 1=$ modelo unifactorial; $\mathrm{M} 2=$ modelo de tres factores; $\chi_{\text {corr }}^{2}=$ chi cuadrado corregido; $\mathrm{GL}=\operatorname{grados}$ de libertad; $p=$ probabilidad; $\mathrm{CFI}_{\text {corr }}=$ Comparative Fit Index corregido; $\mathrm{NFI}_{\text {corr }}=$ Normed Fit Index corregido; NNFI ${ }_{\text {corr }}=$ Non-Normed Fit Index corregido; AIC = Akaike Information Criterion; Dif. $=$ Diferencia

$* * p<0.001$.

Fuente: elaboración propia

(M1) donde todos los ítems cargaban hacia un factor latente, obtuvieron valores por encima de 0.9 para los índices de ajuste relativo $\mathrm{NNFI}_{\text {corr }}$ y $\mathrm{CFI}_{\text {corr }}$ recomendados por Hu y Bentler (1999), excepto el $\mathrm{NFI}_{\text {corr }}$ que obtuvo un valor de 0.87. El modelo de tres factores (vigor, dedicación y absorción) (M2) obtuvo valores por encima de 0.9 en todos los índices de ajuste relativo, por lo que presenta mejor ajuste que el M1. Debido a que los modelos no contienen el mismo número de parámetros y no son modelos anidados, se utilizó el AIC para comparar ambos modelos (Byrne, 2010). Un valor bajo del AIC indica que el modelo posee mejor ajuste. En el presente estudio, el M2 posee un mejor ajuste global en comparación al M1. A la luz de los resultados se concluyó que, en la muestra de estudio, la UWES se ajusta mejor a una estructura de tres factores.

Una vez obtenida la estructura factorial de la UWES, se procedió a examinar la fiabilidad, fiabilidad compuesta, validez convergente y validez discriminante de la versión final de la escala. Los valores alfa de Cronbach fluctuaron entre 0.8 y 0.92. La Tabla 1 resume los valores de fiabilidad compuesta para cada dimensión. La fiabilidad compuesta (FC) de las dimensiones de la escala están por encima de 0.7, recomendado por Raykov y Shrout (2002) (rango 0.81 - 0.87).

Se examinaron la validez convergente y discriminante de la escala siguiendo el procedimiento propuesto por Fornell y Larcker (1981). En cuanto a la validez convergente, se calculó la varianza media extraída (VME), que indica la proporción de la varianza en los ítems explicada por los factores latentes. Los valores de VME de todos los factores estuvieron por encima del 0.5, recomendado por Fornell y Larcker (1981). En cuanto a la validez discriminante, los tres factores no comparten una cantidad sustancial de varianza entre sí. La varianza promedio compartida entre los factores es del 0.57 (rango 0.51-0.64). La varianza compartida entre dos factores de la escala siempre es menor a la varianza explicada por cada uno de los factores (VME), lo cual cumple con el criterio de validez discriminante propuesto por Fornell y Larcker (1981). Esto es indicativo de que todas las subescalas están altamente correlacionadas, pero que cada una mide una dimensión distinta, sin que exista redundancia entre las subescalas del instrumento. Por último, se pasó a realizar un análisis de discriminación de los ítems, a través del índice de correlación ítem total. La Tabla 3 presenta los índices de discriminación, los cuales están por encima del mínimo recomendado de 0.3 (Kline, 2005).

Como resultado del proceso de análisis de los ítems y la estructura factorial con ecuaciones estructurales, las cargas factoriales de cada indicador oscilan entre 0.68 y 0.82 , así como las correlaciones entre los factores oscilan entre 0.72-0.8. Mientras que las correlaciones de las puntuaciones directas de la UWES oscilan entre 0.87 y 0.93.

\section{Discusión}

El objetivo principal de este estudio es analizar las propiedades psicométricas de la versión en español de la UWES-9 en un grupo de personas trabajadoras en Puerto Rico. Los resultados de este estudio aportan nuevo conocimiento sobre la estructura 
TABLA 3

Estadísticos descriptivos y de distribución para los ítems de la versión final de la escala

\begin{tabular}{|c|c|c|c|c|c|c|c|}
\hline Ítem & Media & $\begin{array}{l}\text { Desviación } \\
\text { Estándar }\end{array}$ & $\begin{array}{l}\text { Kolmogorov- } \\
\text { Smirnov }\end{array}$ & $\begin{array}{l}\text { Shapiro- } \\
\text { Wilk }\end{array}$ & $\begin{array}{c}\text { Índice } \\
\text { discriminación }\end{array}$ & $\begin{array}{c}\text { Carga } \\
\text { Factorial }\end{array}$ & $\begin{array}{l}\text { R2 del } \\
\text { ítem }\end{array}$ \\
\hline $\begin{array}{l}\text { 1. En mi trabajo me siento lleno de } \\
\text { energía (V) }\end{array}$ & 4.63 & 1.32 & 0.28 & 0.85 & 0.73 & 0.8 & 0.64 \\
\hline $\begin{array}{l}\text { 2. Soy fuerte y vigoroso en } \mathrm{mi} \\
\text { trabajo }(\mathrm{V})\end{array}$ & 4.89 & 1.21 & 0.26 & 0.82 & 0.7 & 0.75 & 0.56 \\
\hline $\begin{array}{l}\text { 3. Estoy entusiasmado con mi } \\
\text { trabajo (D) }\end{array}$ & 4.65 & 1.45 & 0.25 & 0.83 & 0.8 & 0.89 & 0.79 \\
\hline 4. Mi trabajo me inspira (D) & 4.45 & 1.57 & 0.24 & 0.85 & 0.81 & 0.89 & 0.79 \\
\hline $\begin{array}{l}\text { 5. Cuando me levanto por las } \\
\text { mañanas tengo ganas de ir a } \\
\text { trabajar }(\mathrm{V})\end{array}$ & 4.08 & 1.67 & 0.24 & 0.89 & 0.75 & 0.8 & 0.64 \\
\hline $\begin{array}{l}\text { 6. Soy feliz cuando estoy absorto } \\
\text { en mi trabajo (A) }\end{array}$ & 4.46 & 1.53 & 0.24 & 0.86 & 0.73 & 0.77 & 0.59 \\
\hline $\begin{array}{l}\text { 7. Estoy orgulloso del trabajo que } \\
\text { hago (D) }\end{array}$ & 5.16 & 1.31 & 0.34 & 0.69 & 0.68 & 0.69 & 0.48 \\
\hline $\begin{array}{l}\text { 8. Estoy inmerso en mi trabajo } \\
\text { (A) }\end{array}$ & 4.79 & 1.37 & 0.26 & 0.81 & 0.74 & 0.81 & 0.66 \\
\hline $\begin{array}{l}\text { 9. Me "dejo llevar" por mi trabajo } \\
\text { (A) }\end{array}$ & 4.28 & 1.62 & 0.24 & 0.87 & 0.63 & 0.8 & 0.64 \\
\hline
\end{tabular}

Nota. $\mathrm{V}=$ Vigor; $\mathrm{D}=$ Dedicación; A = Absorción; Grados de libertad de las pruebas Kolmogorov-Smirnov y Shapiro-Wilk = 2.796 y todos sus valores $p<0.001$.

Fuente: elaboración propia

factorial de la UWES, en el contexto laboral latinoamericano. Nuestros hallazgos evidencian que la estructura factorial de la UWES con una muestra de trabajadores en Puerto Rico, consta de tres factores altamente correlacionados, aunque también el modelo unifactorial posee índices de ajuste aceptables, exceptuando el NFI corr $_{\text {. }}$ Al mismo tiempo, la UWES y sus subescalas cuentan una adecuada consistencia interna, lo que es cónsono con los hallazgos de múltiples estudios previos (Salanova et al., 2000; Schaufeli et al., 2006; Seppälä et al., 2009).

Se puede destacar que la solución unifactorial de la UWES resultó aceptable para la medición y evaluación del engagement en el trabajo, aun cuando existe evidencia empírica de estudios anteriores que apoyan la hipótesis de que consta de tres factores altamente correlacionados (Balducci et al., 2010; Salanova et al., 2000; Schaufeli et al., 2002; Schaufeli et al., 2006; Seppälä et al., 2009). No obstante, el modelo factorial con ecuaciones estructurales muestra que la UWES se ajusta mejor al modelo compuesto de vigor, dedicación y absorción, como componentes del engagement. Estos resulta- dos permiten robustecer la vasta evidencia sobre las características del engagement como un constructo multidimensional en un contexto laboral latinoamericano (Puerto Rico). Estas tres subescalas muestran una alta correlación entre sí; sin embargo, los indicadores de validez divergente evidencian que cada subescala examina una dimensión distinta sin producir redundancia. De igual modo, el indicador de la validez convergente muestra que la proporción de varianza de los ítems explicada por los factores latentes es superior al mínimo recomendado de 0.5.

Estos hallazgos tienen implicaciones prácticas sobretodo en el uso de la UWES en contextos de investigación. Dependiendo del propósito de la investigación que se desee realizar, se pueden utilizar las puntuaciones globales de la escala, así como también las puntuaciones de sus subescalas por separado. Las altas correlaciones entre las subescalas para las puntuaciones directas $(0.72-0.8)$ y para los factores latentes $(0.87-0.93)$ pueden ser indicativo de una estructura unidimensional, pero el mejor ajuste de los datos es la estructura de tres factores, lo que provee apoyo sobre tres dimensiones alta- 
mente correlacionadas. Por ejemplo, para efectos de investigación se pueden utilizar las puntuaciones de las subescalas para generar un factor latente de engagement al utilizar ecuaciones estructurales. De otro modo, tal y como señalan Seppälä et al. (2009), si el objetivo es estudiar el engagement en relación con otras variables, es recomendable utilizar una combinación unifactorial del constructo, y si el objetivo del estudio es estudiar los factores del engagement, es recomendable entonces utilizar las tres dimensiones del constructo. Asimismo, resulta una buena práctica, tomando en consideración los resultados de este estudio y de anteriores, utilizar una puntuación única del engagement (modelo unifactorial), al realizar análisis de regresión. Esta práctica ayuda a evitar problemas de multicolinealidad, debido a las altas intercorrelaciones entre los factores.

Los resultados de este trabajo deben ser interpretados de acuerdo a sus limitaciones, sin menospreciar sus aportaciones. Una de sus limitaciones es que los datos provienen de estudios donde se utilizó el muestreo por disponibilidad. Esta limitación no permite la generalización de nuestros resultados a otros contextos de trabajo puertorriqueños. Sin embargo, la composición heterogénea de la muestra, así como la coincidencia de los resultados con otros estudios sobre las propiedades de la UWES, podría acortar esta limitación. Sin embargo, este estudio sí aporta evidencia sobre la estructura multifactorial del engagement, mediante los análisis estadísticos robustos con ecuaciones estructurales.

Entre las futuras líneas de investigación sobre las propiedades psicométricas de la UWES, se podrían desarrollar normas para la baremación de las puntuaciones de la escala. También sería de sumo interés realizar estudios transculturales con diferentes países latinoamericanos sobre las propiedades psicométricas de la UWES, así como poner a prueba su invarianza factorial, tomando en consideración nuestro contexto laboral.

A modo de conclusión, se puede demostrar que la versión en español de la UWES posee adecuadas propiedades psicométricas (validez de constructo y fiabilidad) para ser utilizada, tanto en el ámbito investigativo como en el de la práctica profesional en contextos laborales puertorriqueños. Se espera que la presente investigación incite a otros profesionales a continuar realizando investigaciones sobre el engagement en el trabajo, como parte de una agenda común para el avance de la psicología del trabajo y las organizaciones en Latinoamérica.

\section{Referencias}

Acosta, H., Torrente, P., Llorens, S., \& Salanova, M. (2013). Prácticas organizacionales saludables: un análisis estudio de su impacto relativo sobre el engagement con el trabajo. Revista Peruana de Psicología y Trabajo Social, 2(1), 107-120.

Akaike, H. (1987). Factor analysis and AIC. Psychometrika, 52(3), 317-332.

Andrade, V., \& Gómez, I. C. (2008). Salud laboral: investigaciones realizadas en Colombia. Pensamiento Psicológico, 4(10), 9-25.

Arbuckle, J. L. (2011). Amos ${ }^{T M} 20$ User's Guide. Chicago: SPSS Inc.

Bakker, A. B. (2011). An evidence-based model of work engagement. Current Directions in Psychological Science, 20(4), 265-269.

Bakker, A. B., \& Demerouti, E. (2008). Towards a model of work engagement. The Career Development International, 13(3), 209-223.

Balducci, C., Fraccaroli, F., \& Schaufeli, W. B. (2010). Psychometric properties of the Italian version of the Utrecht Work Engagement Scale (UWES-9): A cross-cultural analysis. European Journal of Psychological Assessment, 26(2), 143-149.

Byrne, B. M. (2010). Structural equation modeling with AMOS: Basic concepts, applications, and programming. New York: Psychology Press.

Cole, M. S., Walter, F., Bedeian, A. G., \& O'Boyle, E. H. (2012). Job burnout and employee engagement a meta-analytic examination of construct proliferation. Journal of Management, 38(5), 1550-1581.

Demerouti, E., \& Bakker, A. B. (2008). The Oldenburg Burnout Inventory: A good alternative to measure burnout and engagement. En J. Halbesleben (Ed.), Stress and burnout in health care (pp. 65-78). Hauppauge, NY: Nova Sciences Publishers.

Demerouti, E., Bakker, A. B., Nachreiner, F., \& Schaufeli, W. B. (2001). The job demands-resources model 
of burnout. Journal of Applied Psychology, 86(3), 499-512.

Demerouti, E., Mostert, K., \& Bakker, A. B. (2010). Burnout and work engagement: A thorough investigation of the independency of both constructs. Journal of Occupational Health Psychology, 15(3), 209-222.

Fong, T.C., \& Ng, S. (2012). Measuring engagement at work: Validation of the Chinese version of the Utrecht Work Engagement Scale. International Journal of Behavioral Medicine, 19(3), 391-397.

Fornell, C., \& Larcker, D. F. (1981). Evaluating structural equation models with unobservable variables and measurement error. Journal of Marketing Research, 18(1), 39-50.

Gómez, I. C. (2007). Salud laboral: una revisión a la luz de las nuevas condiciones de trabajo. Universitas Psychologica, 6(1), 105-113.

González-Romá, V., Schaufeli, W. B., Bakker, A. B., \& Lloret, S. (2006). Burnout and work engagement: Independent factors or opposite poles? Journal of Vocational Behavior, 68(1), 165-174.

Hakanen, J. J., Schaufeli, W. B., \& Ahola, K. (2008). The job demands-resources model: A three-year cross-lagged study of burnout, depression, commitment, and work engagement. Work $\mathcal{E}$ Stress, 22(3), 224-241.

Halbesleben, J. R. B., \& Demerouti, E. (2005). The construct validity of an alternative measure of burnout: Investigating the English translation of the Oldenburg Burnout Inventory. Work E Stress, 19, 208-220.

Hallberg, U. E., \& Schaufeli, W. B. (2006). 'Same Same' But Different? Can Work Engagement Be Discriminated from Job Involvement and Organizational Commitment? European Psychologist, 11(2), 119-127.

Hu, L. T., \& Bentler, P. M. (1999). Cutoff criteria for fit indexes in covariance structure analysis: Conventional criteria versus new alternatives. Structural Equation Modeling: A Multidisciplinary Journal, 6(1), 1-55.

Kahn, W. A. (1990). Psychological conditions of personal engagement and disengagement at work. Academy of Management Journal, 33(4), 692-724.
Kim, W., Kolb, J. A., Kim, T. (2013). The relationship between work engagement and performance a review of empirical literature and a proposed research agenda. Human Resource Development Review, 12(3), 248-276.

Kline, T. J. B. (2005). Psychological testing: A practical approach to design and evaluation. Thousand Oaks: Sage.

Llorens, S., Bakker, A. B., Schaufeli, W., \& Salanova, M. (2006). Testing the robustness of the job demandsresources model. International Journal of Stress Management, 13(3), 378-391.

Lorente, L., Salanova, M., Martínez, I., \& Schaufeli, W. B. (2008). Extension of the job demands-resources model in the prediction of burnout and engagement among teachers over time. Psicothema, 20(3), 354-360.

Mardia, K. V. (1970). Measures of multivariate skewness and kurtosis with applications. Biometrika, 57(3), 519-530.

Martínez-Lugo, M. (2009). La vinculación psicológica en el trabajo (engagement), el síndrome de quemarse por el trabajo y el clima organizacional en un grupo de empleados en Puerto Rico. Revista Interamericana de Psicología Ocupacional, 28(1), 7-15.

Martínez-Lugo, M. E., Rodríguez, A. T., Segarra, B., Donato, M., Rabelo, I., \& Ortiz, L. (2011). El engagement en el trabajo y su relación con la implicación laboral y la adicción al trabajo en un grupo de empleados en Puerto Rico. Ciencias de la Conducta, 26(1), 131-130.

Maslach, C., Jackson, S. E., \& Leiter, M. P. (1996). Maslach Burnout Inventory Manual (3.a ed.). Palo Alto, CA: Consulting Psychologists Press.

Maslach, C., \& Leiter, M. P. (1997). The truth about burnout: How organizations cause personal stress and what to do about it. San Francisco: Jossey-Bass.

Maslach, C., Schaufeli, W. B. \& Leiter, M. P. (2001). Job burnout. Annual Review of Psychology, 52, 397-422.

Mills, M., Culbertson, S., \& Fullagar, C. (2011). Conceptualizing and measuring engagement: An analysis of the Utrecht Work Engagement Scale [Versión electronic]. Journal of Happiness Studies, 13(3), 1-27. http://dx.doi.org/10.1007/s10902-011-9277-3

Muilenburg-Treviño, E. (2009). A psychometric study of work engagement in an American sample (Tesis 


\section{ANÁLISIS DE LAS PROPIEDADES PSICOMÉTRICAS DE LA UTRECHT Work ENGAGEMENT Scale en una muestra de trabajadores en Puerto Rico}

doctoral en Filosofía inédita). Oklahoma State University Oklahoma.

Nerstad, C. G. L., Richardsen, A. M., \& Martinussen, M. (2010). Factorial validity of the Utrecht Work Engagement Scale (UWES) across occupational groups in Norway. Scandanavian Journal of Psycho$\log y, 51(4), 326-333$.

Raykov, T., \& Shrout, P. E. (2002). Reliability of scales with general structure: Point and interval estimation using a structural equation modeling approach. Structural Equation Modeling: A Multidisciplinary Journal, 9(2), 195-212.

Rodríguez Montalbán, R. (2011). Análisis de las propiedades psicométricas del Utrecht Work Engagement Scale en un grupo de empleados en Puerto Rico. Disertación doctoral no publicada. Programa de Psicología Industrial/Organizacional, Universidad Carlos Albizu. San Juan: Puerto Rico.

Rodríguez-Montalbán R, Vélez-Pastrana, M., \& Meléndez, M. (2012). El engagement en el trabajo y la interacción familia-trabajo: un estudio exploratorio. Revista Interamericana de Psicología Ocupacional, 31(2), 73-84.

Román, J. P, Battistelli, A., \& Odoardi, C. (2013). Work engagement as mediator between perceived participation, supervisor support and altruistic behaviors: Empirical results from the Italian social enterprise sector. Universitas Psychologica, 12(3), 899-909.

Salanova, M., \& Schaufeli, W. (2009). El "engagement" en el trabajo: cuando el trabajo se convierte en pasión. Madrid: Alianza.

Salanova, M., Agut, S., \& Peiró, J. (2005). Linking organizational resources and work engagement to employee performance and customer loyalty: The mediation of service climate. Journal of Applied Psychology, 90(6), 1217-1227.

Salanova, M., Llorens, S., Cifre, E., \& Martínez, I. M. (2012). We need a Hero! Toward a validation of the Healthy and Resilient Organization (HERO) Model. Group E Organization Management, 37(6), 785-822.

Salanova, M., Lorente, L., Chambel, J., \& Martínez, I. M. (2011). Linking transformational leadership to nurses' extra-role performance: The mediating role of self-efficacy and work engagement. Journal of Advanced Nursing, 67(9), 2256-2266.

Salanova, M., Martínez, I. M., \& Llorens, S. (2005). Psicología organizacional positiva. En F. Palaci (Ed.), Psicología de la organización (pp. 349-376). Madrid: Prentice Hall.

Salanova, M., Schaufeli, W. B., Llorens, S., Peiro, J. M., \& Grau, R. (2000). Desde el "burnout" al "engagement": iuna nueva perspectiva? Revista de Psicología del Trabajo y de las Organizaciones, 16(2), 117-134.

Sanín, J. A., \& Salanova, M. (2014). Satisfacción laboral: el camino entre el crecimiento psicológico y el desempeño laboral. Universitas Psychologica, 13(1).

Satorra, A., \& Bentler, P. M. (2001). A scaled difference chi-square test statistic for moment structure analysis. Psychometrika, 66(4), 507-514.

Schaufeli, W. B., Bakker, A. B., \& Salanova, M. (2006). The measurement of work engagement with a short questionnaire: A cross-national study. Educational and Psychological Measurement, 66(4), 701-716.

Schaufeli, W. B., Martínez, I., Marques-Pinto, A., Salanova, M., \& Bakker, A. B. (2002). Burnout and engagement in university students: A crossnational study. Journal of Cross-Cultural Psychology, 33(5), 464-481.

Schaufeli, W. B., Salanova, M., González-Romá, V., \& Bakker, A. B. (2002). The measurement of engagement and burnout: A two sample confirmatory factor analytic approach. Journal of Happiness Studies, 3(1), 71-92.

Seligman, M. E. P., \& Csikszentmihalyi, M. (2000). Positive psychology: An introduction. American Psychologist, 55(1), 5-14.

Seppälä, P., Mauno, S., Feldt, T., Hakanen, J., Kinnunen, U., Tolvanen, A., \& Schaufeli, W. (2009). The construct validity of the Utrecht Work Engagement Scale: Multisample and longitudinal evidence. Journal of Happiness Studies, 10(4), 459-481. Shimazu, A. A., Schaufeli, W. B., Kosugi, S. S., Suzuki, A. A., Nashiwa, H. H., Kato, A. A., \& ... Goto, R. R. (2008). Work engagement in Japan: Validation of the Japanese version of the Utrecht Work Engagement Scale. Applied Psychology: An International Review, 57(3), 510-523. 
Sonnentag, S. (2003). Recovery, work engagement, and proactive behavior: A new look at the interface between non-work and work. Journal of Applied Psychology, 88(3), 518-528.

Spontón, C., Medrano, L. A., Maffei, L., Spontón, M., \& Castellano, E. (2012). Validación del cuestionario de Engagement UWES a la población de trabajadores de Córdoba, Argentina. Liberabit, 18(2), 147-154.

Storm, K., \& Rothmann, S. (2003). A psychometric analysis of the Utrecht Work Engagement Scale in the South African police service. SA Journal of Industrial Psychology, 29(4), 62-70.
Torrente, P., Salanova, M., Llorens, S., \& Schaufeli, W. B. (2012). Teams make it work: How team work engagement mediates between social resources and performance in teams. Psicothema, 24(1), 106-112.

Wefald, A. J., \& Downey, R. G. (2009). Construct dimensionality of engagement and its relation with satisfaction. Journal of Psychology: Interdisciplinary and Applied, 143(1), 91-111.

Xanthopoulou, D., Bakker, A. B., Demerouti, E., \& Schaufeli, W. B. (2009). Work engagement and financial returns: A diary study on the role of job and personal resources. Journal of Occupational and Organizational Psychology, 82(1), 183-200. 